\title{
Peak-Seeking Optimization of Spanwise Lift Distribution for Wings in Formation Flight
}

AIAA Guidance, Navigation, and Control Conference Minneapolis, MN August 14, 2012 


\section{Roadmap to Formation Flight in the NAS}

$10 \%$ Power Reduction (German Institute for Fluid Mechanics)

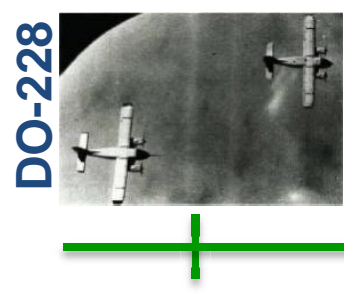

1995

Relative Navigation and Formation Autopilot Systems

헝 Understanding Impacts on Structures, Engines, Actuators and Ride Quality

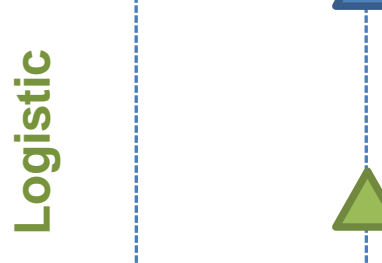

言

Minimum Separation Requirement Exceptions

Avionics and Airframe Certification

Pilot Training

TRL: 1

14\% Fuel Savings 9\% Fuel Savings (NASA DFRC) (USAF TPS)
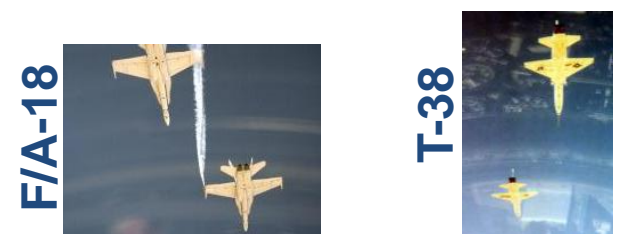

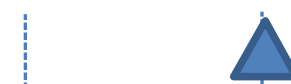

Probabilistic Vortex Models

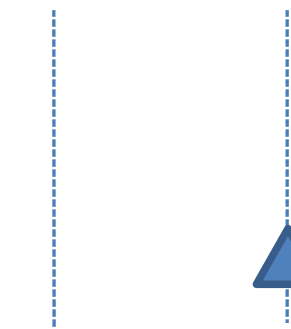

Formation Flight Capabilities in COTS Avionics

2

3

6

-8\% Fuel Savings (NASA CAPFIRE)

$\frac{N}{0}$ Operational Concept Development

Tools for Formation Routing and Scheduling

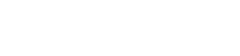

5
C-17 SAVE (DARPA / AFRL / Boeing)

Fall 2012

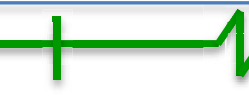

2012
Commercial Formation Flight Demonstration In the NAS

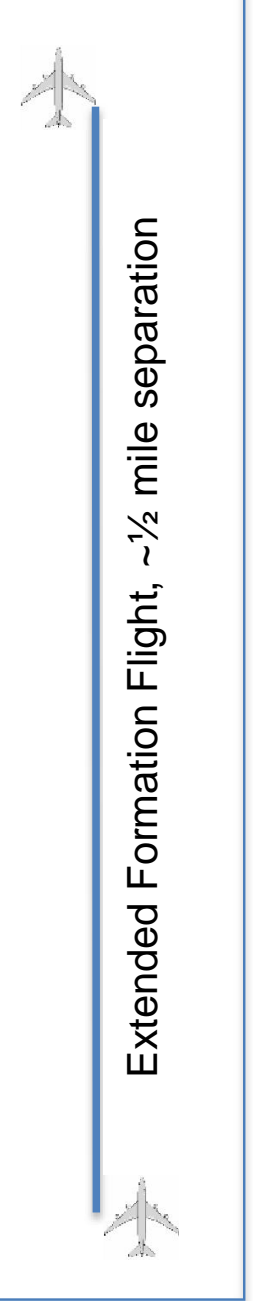




\section{Formation Flight Aerodynamics}

口 In-trail flight within another aircraft's wingtip vortex upwash field can lower induced drag
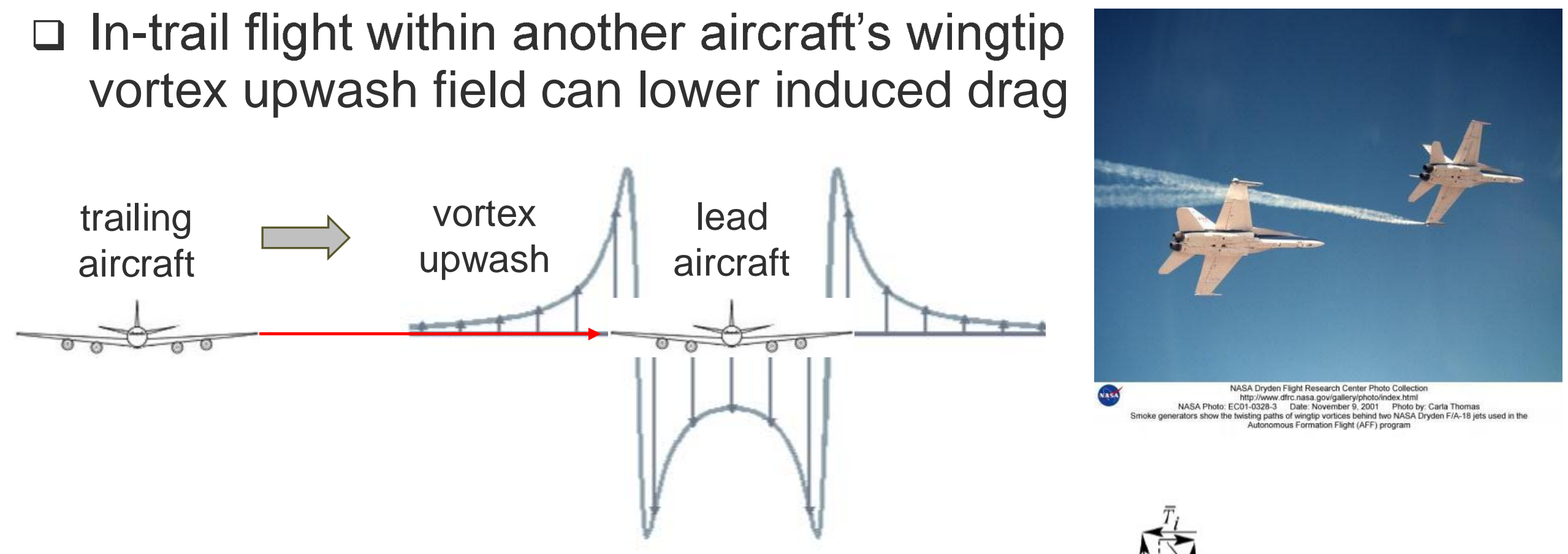

$\square$ The primary effects of flight within the wingtip vortex upwash are:

- forward rotation of the lift vector, lowering induced drag

- asymmetric span-wise lift distribution, causing roll trim imbalance

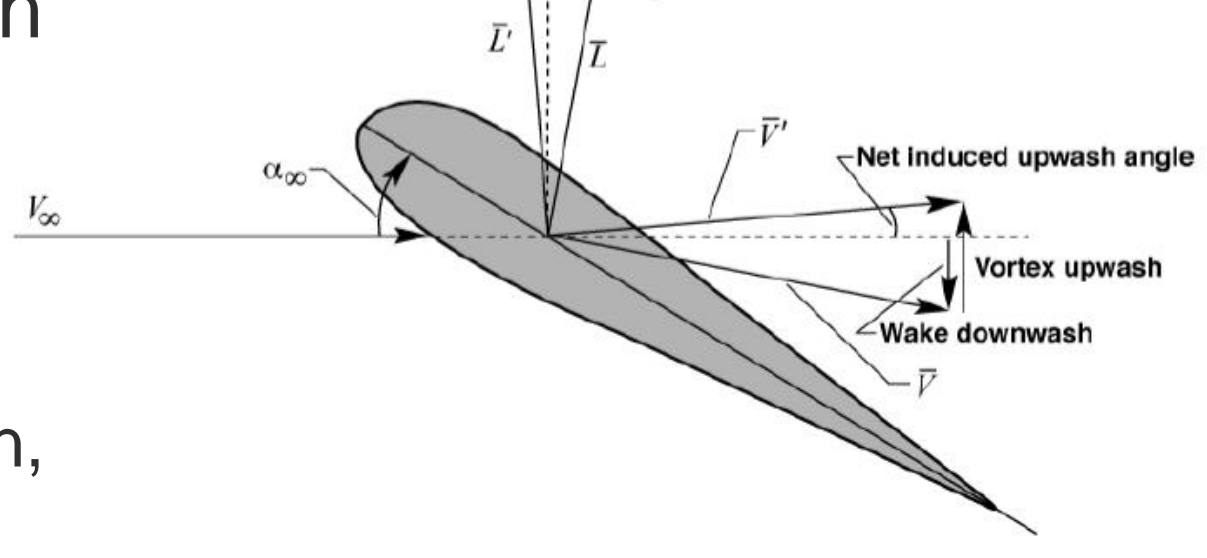




\section{Formation Flight Optimization}

Real-time determination of optimal relative position to the lead aircraft

- lateral and vertical drift of the vortex field

口 uncertainty regarding the best location within the upwash field

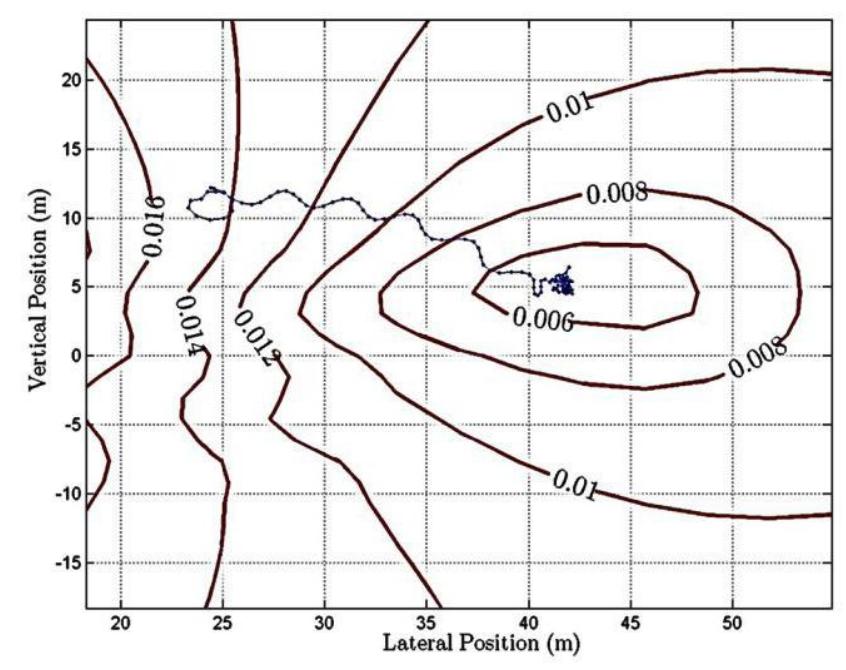

口 Ryan and Speyer, ACC (2010)
Real-time determination of the optimal roll trim solution

- traditional anti-symmetric roll trim using ailerons can lower the effectiveness of formation flight

- optimal deployment of trailingedge wing surfaces can balance induced rolling moments and enhance drag reduction

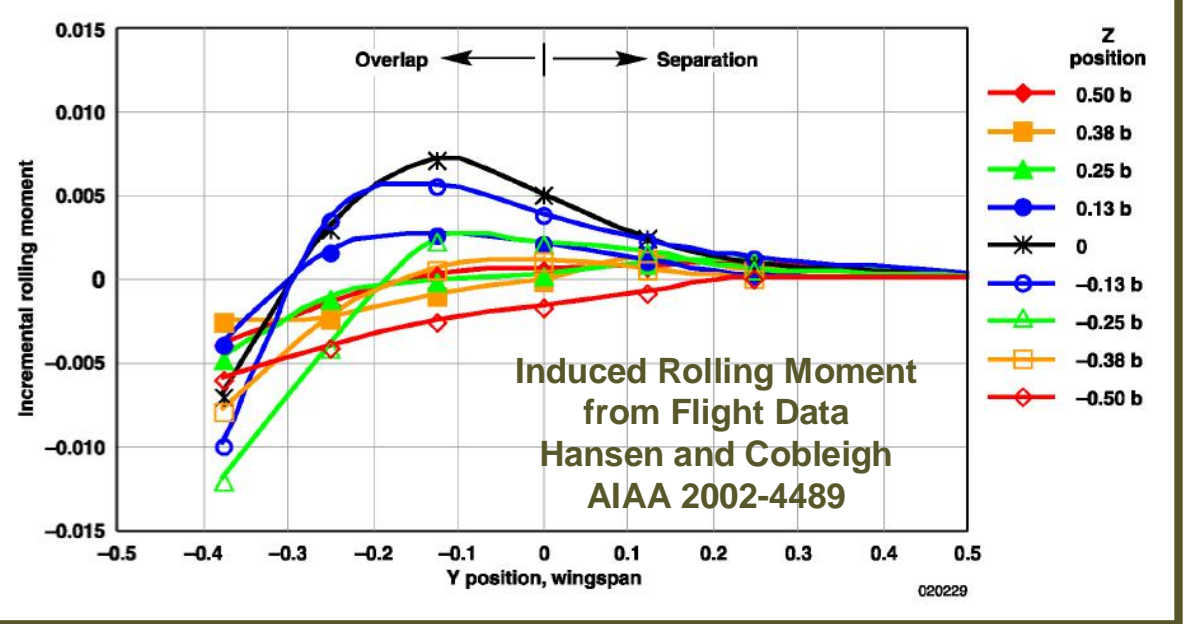




\section{$\underline{\text { Roll Trim Optimization }}$}

- Analysis of flexible unswept wings has shown that increased wing twist (leading-edge-up) corresponds to greater drag reduction in formation

- Trailing-edge control surfaces can be re-trimmed to alter the spanwise lift distribution and further reduce drag in an asymmetric upwash field
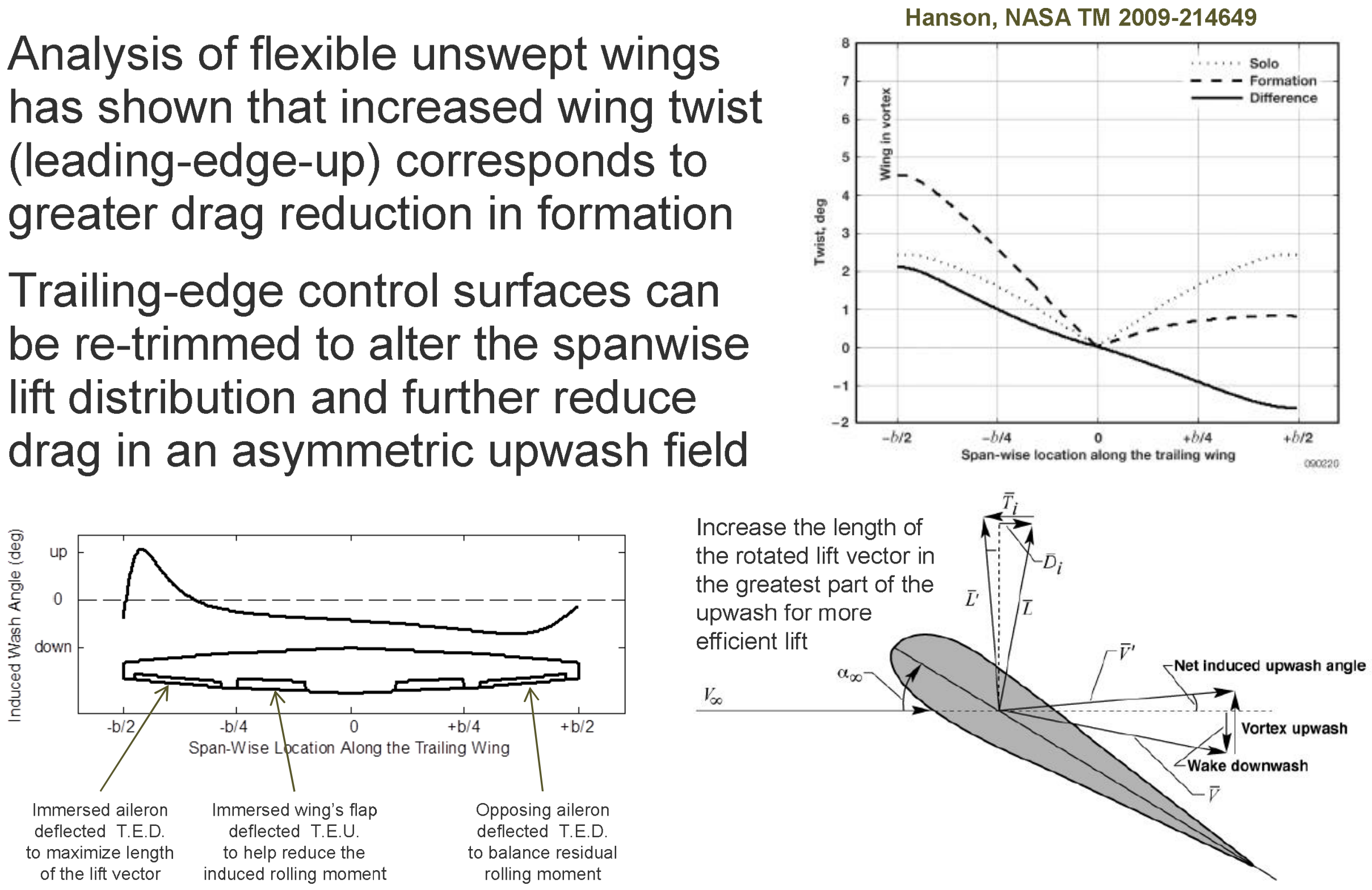


\section{Peak-Seeking Algorithm}

Approximation to the cost function Drag vs. Immersed Aileron Command

$$
\Delta J_{k}=\Delta \delta_{k}^{i b} b_{J}+\frac{1}{2}\left(\Delta \delta_{k}^{i b}\right)^{2} m_{J} \quad \text { where } \quad b_{J}=\frac{\partial J}{\partial \delta^{i b}}, \quad m_{J}=\frac{\partial^{2} J}{\partial \delta^{i b^{2}}}
$$

Multi-Sample Implementation

$$
\Delta J_{k}=H_{k} \zeta_{k}+r_{k} \quad\left\{\Delta J_{k}\right\}=\left[\begin{array}{c}
\Delta J_{k, 1} \\
\Delta J_{k, 2} \\
\vdots \\
\Delta J_{k, N}
\end{array}\right] \quad\left\{H_{k}\right\}=\left[\begin{array}{cc}
\delta_{k, 1}^{i b} & \frac{1}{2}\left(\Delta \delta_{k, 1}^{i b}\right)^{2} \\
\delta_{k, 2}^{i b} & \frac{1}{2}\left(\Delta \delta_{k, 2}^{i b}\right)^{2} \\
\vdots & \vdots \\
\delta_{k, N}^{i b} & \frac{1}{2}\left(\Delta \delta_{k, N}^{i b}\right)^{2}
\end{array}\right] \quad \zeta_{k}=\left[\begin{array}{c}
b_{J} \\
m_{J}
\end{array}\right]
$$

Kalman Filter Estimate of Gradient and Curvature

$$
\zeta_{k}=\Phi \zeta_{k-1}+q_{k-1}
$$

Newton-Raphson Command Update

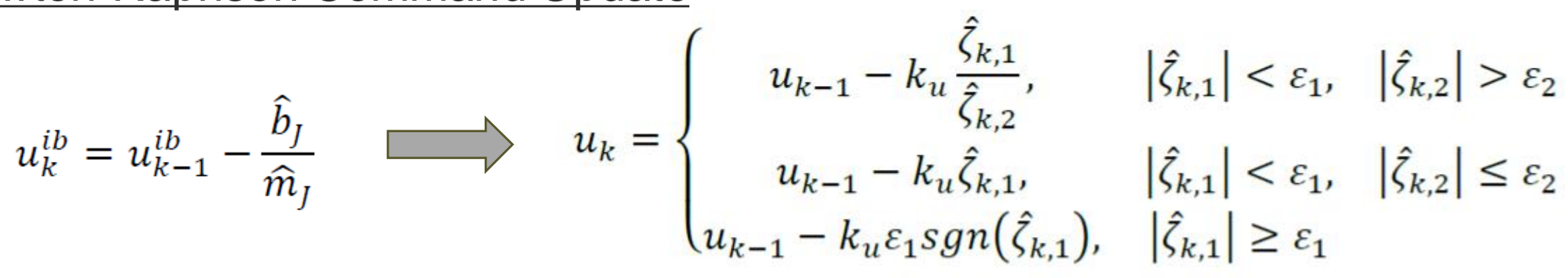




\section{Evaluation}

口 Aerodynamic Modeling

- Horseshoe vortex models

- Vortex strength calculated using the Kutta-Joukowski theorem

$$
\Gamma \rho V b \frac{\pi}{4}=m g
$$

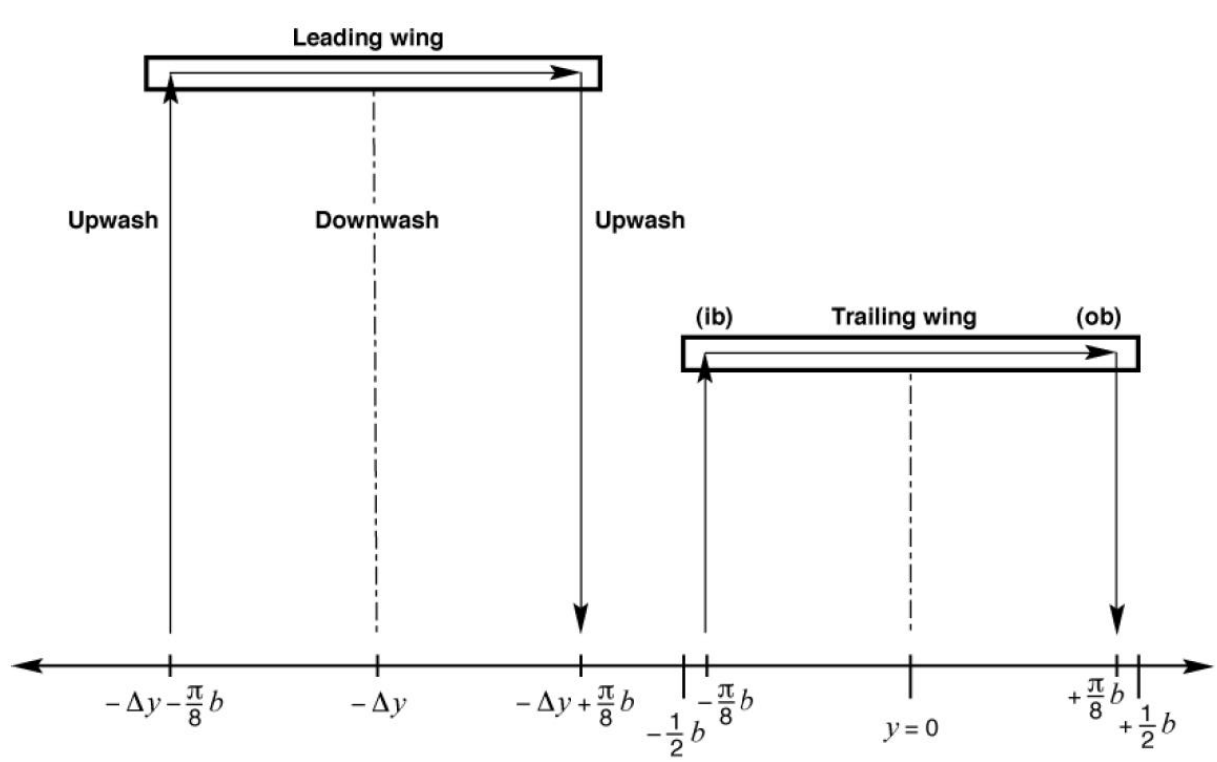

- Induced angle-of-attack calculated from the Burnham-Hallock tangential velocity profile

$$
\alpha_{i}(y)=\frac{\Gamma}{2 \pi V}\left[\frac{\Delta y-\frac{\pi}{8} b+y}{\left(\Delta y-\frac{\pi}{8} b+y\right)^{2}+r_{c}^{2}}-\frac{\Delta y+\frac{\pi}{8} b+y}{\left(\Delta y+\frac{\pi}{8} b+y\right)^{2}+r_{c}^{2}}\right]
$$

Assumption control surface drag is a function of the square of the deflection

$\square$ Control Mixing

- Immersed aileron and flap commanded to equal but opposite deflections

- Opposing aileron deflected as required to maintain roll trim 


\section{$\underline{\text { Results }}$}

- The peak-seeking algorithm located the drag-optimal trim solution

- relative positions, orientations between the aircraft were fixed

- trim surfaces were initialized to non-optimal values

- trim commands were initialized to move the surfaces in the wrong direction

- noise was applied to both measurements and commands

口 Incremental reduction in total drag due to trim modification was approximately $2 \%$
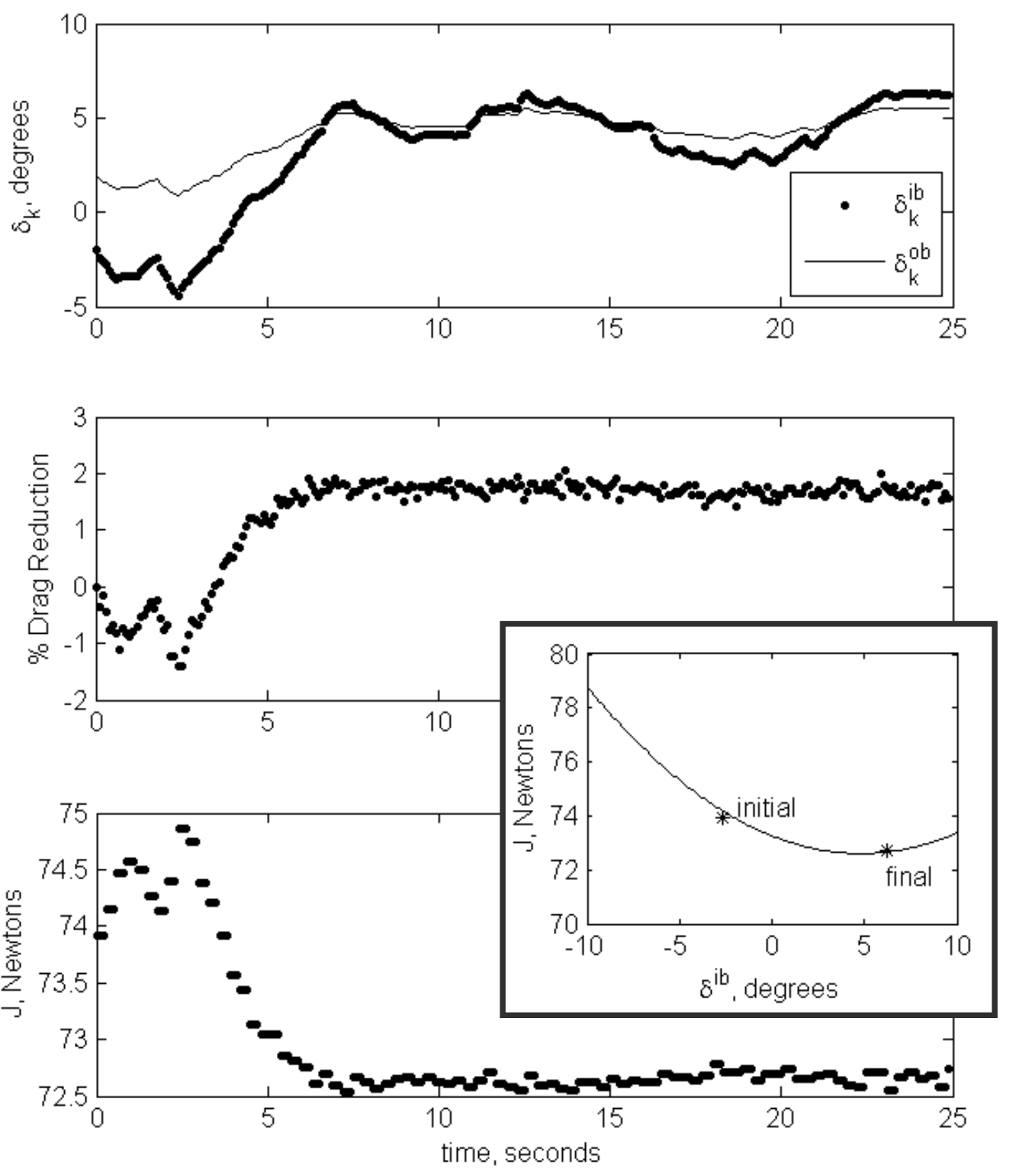


\section{Summary}

口 Unique trim scheme for flight within asymmetric upwash fields

- Traditional anti-symmetric aileron trim was found to reduce the effectiveness of formation flight for drag reduction

- An alternate roll trim scheme was found to improve performance

- A peak-seeking control algorithm was implemented to determine the optimal trim deflections in real-time

口 Future Work

- Confirm results with CFD analysis (in progress)

- Remove the restriction of equal but opposite deflections for the immersed aileron and flap (increases dimensionality of the problem)

- Integrate with a position-optimizing peak-seeking algorithm

- Evaluate in a full 6DOF simulation

- Confirm results through flight research 
NASA 